\title{
Utilization of KNApSAcK Family Databases for Developing Herbal Medicine Systems
}

\author{
Sony Hartono Wijaya ${ }^{1,2}$, Yuki Tanaka ${ }^{1}$, Md. Altaf-Ul-Amin ${ }^{1}$, Aki Hirai \\ Morita $^{1}$, Farit Mochamad Afendi ${ }^{3}$, Irmanida Batubara ${ }^{4}$, Naoaki Ono ${ }^{1}$, \\ Latifah K. Darusman ${ }^{4}$, Shigehiko Kanaya ${ }^{1 *}$ \\ ${ }^{(1)}$ Graduate School of Information Science, Nara Institute of Science and Technology, 8916-5 Takayama, \\ Ikoma, Nara 630-0192, Japan \\ ${ }^{(2)}$ Department of Computer Science, Bogor Agricultural University, Jl. Meranti Kampus IPB Dramaga, \\ Bogor 16680, Indonesia \\ ${ }^{(3)}$ Department of Statistics, Bogor Agricultural University, Jl. Meranti Kampus IPB Dramaga, Bogor \\ 16680, Indonesia \\ ${ }^{(4)}$ Biopharmaca Research Center, Bogor Agricultural University, Kampus IPB Taman Kencana, Jl. \\ Taman Kencana No. 2, Bogor 16128, Indonesia
}

(Received March 11, 2016; Accepted May 17, 2016)

\begin{abstract}
Recently, the use of traditional medicines for medical treatment and maintaining good health is increasing. This condition has been followed by the increasing number of research activities, publications and databases of crude drug systems to support the scientific aspects of traditional medicines. Nevertheless, the information about traditional medicines is scattered in an unorganized manner and the existing traditional medicine databases have been stored by different database schemas. Therefore, a standardized tool that integrates and provides information about various herbal medicines is needed. In this study, we developed a mobile application with Waterfall Method, called as Herbal Medicine Systems, for various kinds of crude drug systems as a reference of traditional medicines. The KNApSAcK Family Databases were used as a role model to design the database schemas of crude drug systems by using data warehouse pre-processing technique. The Herbal Medicine Systems application is preloaded with 336 and 5,310 Kampo and Jamu formulas respectively from the KNApSAcK Kampo and KNApSAcK Jamu databases. In addition, the Herbal Medicine Systems can predict the efficacy of crude drug combinations by using Random Forest classifier developed based on the formulation of Indonesian Jamu with the average accuracy of $90 \%$.
\end{abstract}

Keywords: traditional medicines, mobile application, crude drugs, Jamu, Kampo, Random Forest

*skanaya@gtc.naist.jp 


\section{Introduction}

The number of medicinal plants is estimated to be 40,000 to 70,000 around the world [1]. Many countries utilize these plants as blended herbal medicines for treatment of diseases or maintaining people in a good health [2,3], e.g., traditional Chinese medicine (TCM), Japanese Kampo medicine, Indonesian Jamu, and Ayurveda, Siddha and Unani in the South Asia. In the last decade, globally there has been an increasing trend in the use of herbal medicines. According to the Secretariat of the Convention on Biological Diversity, global sales of total herbal products were estimated to reach US\$ 60,000 million in 2000 (WHO 2003). It shows that many people in the world moving towards using herbal products in their daily life. Nevertheless, the availability of information about traditional medicines is scattered in an unorganized manner. It makes difficult for the consumers to obtain and explore supporting information about herbal medicines.

Furthermore, this trend has been followed by the increasing number of research activities, publications and development of databases of crude drug systems to support the scientific aspects of traditional medicines. Many researchers have systemized the formulations and developed basic scientific principles of herbal medicines, i.e. Kampo [4,5], Jamu [6-8], TCM [9,10], Ayurveda $[11,12]$, etc. In accordance with their findings, they need a tool to disseminate their research results so that it can be utilized by public. Moreover, the existing traditional medicine databases have been recorded by different database schemas making it difficult to integrate and to manage the data from various crude drug systems. Therefore, a standardized tool that integrates and provides information about various herbal medicines is needed. In order to obtain high quality software products, a proper software development process is required [13-15]. One of software engineering methods is the Waterfall method, which is widely used and well known with many advantages, i.e. this method is suitable for application development where the requirements are well defined [16,17].

The KNApSAcK Family Databases have been developed to systematize the crude drug systems by providing information about species-metabolite relations and the medicinal usage of plants based on traditional and modern knowledge [18]. The KNApSAcK Kampo and KNApSAcK Jamu are members of KNApSAcK Family Databases that comprehensively explain about Japanese Kampo and Indonesian Jamu medicines. These databases present information about selected herbal ingredients, omics information of plants and humans, and physiological activities in humans.

In Indonesia, a Jamu medicine is made by mixture of several plants. The combination of plants used as Jamu ingredients determines the efficacy of a Jamu formula.
Therefore, it may be useful to model the ingredients of Jamu and use this model to predict the efficacy of Jamu formulas. Random Forest (RF), an ensemble method that consists of many decision trees, can be used to generate the efficacy prediction model. RF builds the decision trees using a bootstrap sample of the data, and at each split the candidate set of variables is a random subset of the variables. RF has many advantages and also outperforms other classification methods, i.e. k-Nearest Neighbor (KNN) and Support Vector Machines (SVM) $[19,20]$.

This study is intended to develop an application with Waterfall Method for exploring various herbal medicines using Android platform. Android has been chosen because this platform has led the market share in mobile application downloads since 2011 [21]. In addition, it is hard to find herbal medicine applications in their store, especially Japanese Kampo and Indonesian Jamu. RF has been chosen as a classifier in order to generate the model for efficacy prediction of a combination of crude drugs.

\section{Methods}

We developed a mobile application, called Herbal Medicine Systems (HMS), for exploring various kinds of crude drug systems and for disseminating research findings. The Waterfall method was used as a guideline to develop the HMS application and Random Forest classifier was used to generate the model for efficacy prediction of crude drug combinations based on the formulation of Indonesian Jamu.

\section{a. Development of Herbal Medicine Systems}

The development of HMS with the Waterfall method mainly consists of five phases as follows: requirements definition, system and software design, implementation and unit testing, integration and system testing, and operation and maintenance (Fig. 1) [13]. The Unified Modeling Language (UML) ver. 2.0 was used in the early stages of the Waterfall Model as a modeling language [22]. In the implementation phase, Android Studio [23,24] and SQLiteStudio [25] were used to develop the mobile application and to develop the database of crude drug systems respectively. For testing, we applied Black-box testing to examine the functionality of HMS application. Then, the resulted application was deposited at Google Play Store as freely downloadable. 


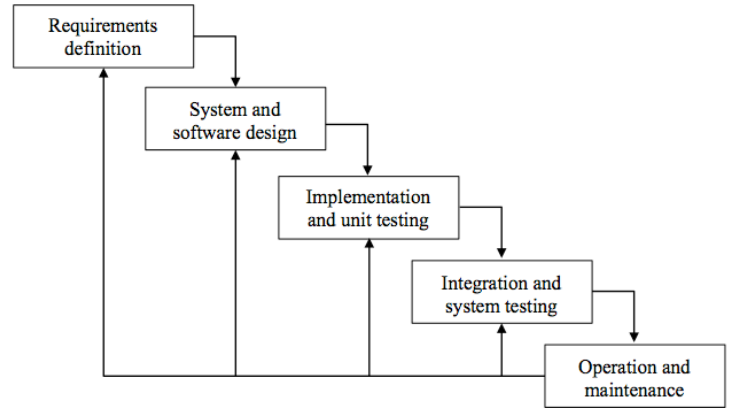

Fig. 1. The Waterfall Method (Sommerville 2010)

Fig. 2 shows a schematic illustration of HMS development. The KNApSAcK Family Databases allow free use and share of data. KNApSAcK Kampo and KNApSAcK Jamu, was utilized to design the database schemas as a role model of crude drug systems by using data warehouse pre-processing technique [26]. Data warehousing combines the data from multiple databases into HMS database. All data from various databases that we intended to integrate were extracted, cleaned, transformed, loaded, and refreshed. A data warehouse pulls all the data from the various data sources, converts all the data into a common format so that one set of data is compatible with another, and loads this new data into HMS database.

\section{b. Random Forest classifier}

Random Forest algorithm mainly includes four steps as follows: bootstrap resampling, random feature selection, full depth decision tree growing, and out-of-bag (OOB) error estimation [27,28]. Given a set of $N$ binary labeled training samples $\left\{x_{i}, y_{i}\right\}, i=1$ to $\mathrm{N}$, where $x_{i}$ is a vector of predictor variables (features) and $y_{i}$ is the response variable (class label). A random forest is constructed by generating ntree decision trees from these samples. For each tree, the same number of $N$ samples is randomly selected with replacement (bootstrap resampling) to form a new training set, and the samples not selected are called the OOB samples.
Using this new training set, a decision tree is grown to the largest extent possible without any pruning according to the classification and regression tree (CART) methodology [29]. For the split of each node, only a small number of mtry randomly selected features are considered (random feature selection). This algorithm estimates an OOB error in the process of constructing the forest. With the construction of a decision tree, each OOB sample is tested, and its OOB classification result is collected. Upon the completion of the construction of the entire forest, OOB classification results for each sample are used to determine a decision for this sample via a majority-voting rule. The fraction of decisions that disagree with the true class label is called as the OOB error.

\section{Results and discussion}

We followed the Waterfall method to develop the HMS application. Then, the efficacy prediction of crude drug combinations can be performed based on the classification algorithm of Random Forest.

\section{a. Requirements definition}

We expect that HMS becomes a major and popular application for exploring various crude drug medicines and most of the HMS features can be run offline using common database format. Initially, we defined and captured the functional requirements of HMS by examining KNApSAcK Kampo and KNApSAcK Jamu. Next, we modeled the interactions between user and HMS by utilizing use case diagram. Fig. 3 shows the use case diagram of HMS, which encompasses one actor and 13 use cases [22]. The HMS mainly consists of four features, i.e. find formulas/recipes based on their name or efficacy, find crude drugs based on their name or effect, analyze herbal medicines, and maintain the database of HMS. The user of HMS will input a query to

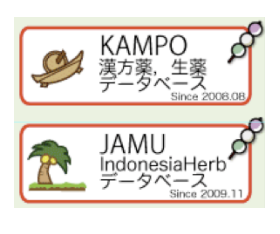

The Waterfall Model (Sommerville 2010)
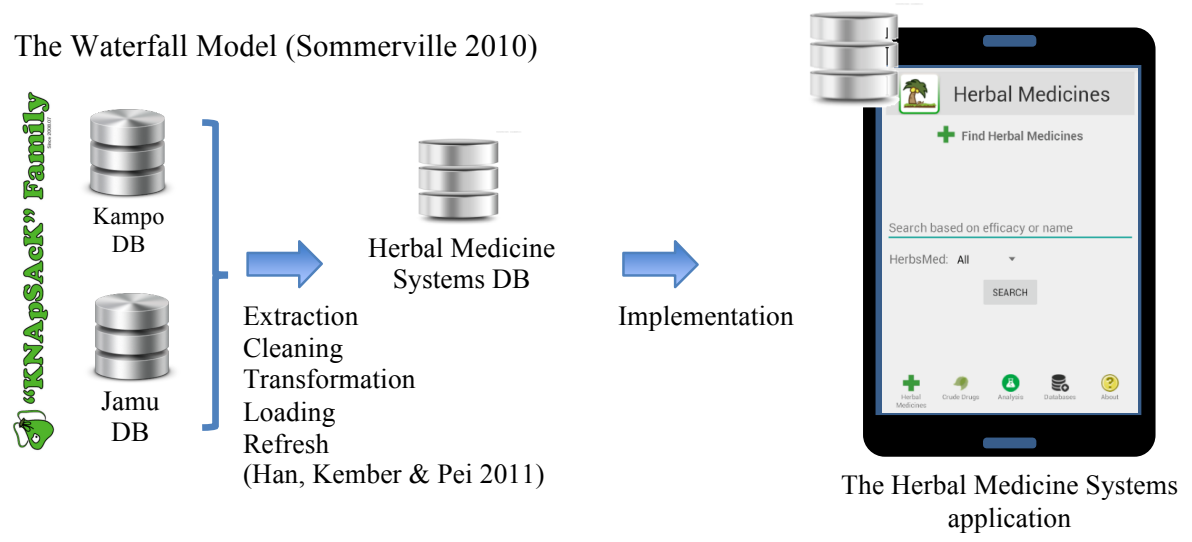

Fig. 2. Illustration of Herbal Medicine Systems development 
obtain their expected information or interact with the application by touching the menu buttons. The analysis of herbal medicines menu covers comparison between herbal medicine formulas and efficacy prediction of a combination of Jamu ingredients. Furthermore, the HMS application can be used for modifying and cross-searching various kinds of crude drug systems.

\section{b. System and software design}

The database and application of HMS were designed by elaborating the functional requirements. Initially, the database structure was developed by implementing data warehouse pre-processing technique (Fig. 2). We used Kampo and Jamu databases to design a common structure of database of Herbal Medicine Systems. Those databases were collected from the KNApSAcK Family databases (http://kanaya.naist.jp/KNApSAcK_Family/). Both Kampo and Jamu databases consist of four tables: gakumei (scientific name), haigo (combination), Kampo/Jamu, and syouyaku (raw drug). Each table is composed of various columns, where each column indicates an attribute. The attributes of every table in the Kampo database are as follows: gakumei (gname, sid), haigou (fid, kid, reference, sid_g), Kampo (kid, kname_j, kname, kname_k, effect), and syouyaku (sid, sname_j, sname_k, name, gname, effect, comment, reference). On the other hand, the attributes of every table in the Jamu database are as follows: gakumei (gname, sid), haigou (fid, jid, company, brand, sid_g), Jamu (jid, cname, jname, effect, effectgroup, jamusource) and syouyaku (sid, sname, iname, ename, gname, position, effect, comment, reference).

We analyzed and extracted important attributes from the Kampo and Jamu databases of the KNApSAcK database systems, related to aforementioned use case in the requirements definition step, i.e. we obtained all information related to finding crude drug use case in the syouyaku table of Kampo and Jamu databases. Furthermore, we designed the class diagram to develop the database of Herbal Medicine Systems [22]. Fig. 4 indicates the HMS domain model class diagram. The HMS class diagram consists of nine classes, i.e. type of herbal medicines (MedType), efficacy groups (DClass), list of herbal medicines (HerbsMed), Company, reference of used formulas (Refformula), Combination, list of crude drugs (CrudeDrug), Plant, and relationship between plant and crude drugs (Plant-Crude).

Kampo and Jamu formulas were considered as preloaded herbal medicines in the HMS application. Each Jamu has only one formula and one Kampo might consist of more than one formula. Between 336 Kampo formulas and 5,310 Jamu formulas from KNApSAcK Family Databases, only 234 Kampo and 3,027 Jamu have information related to their efficacies. In total, the data with efficacy information is composed by 1,023

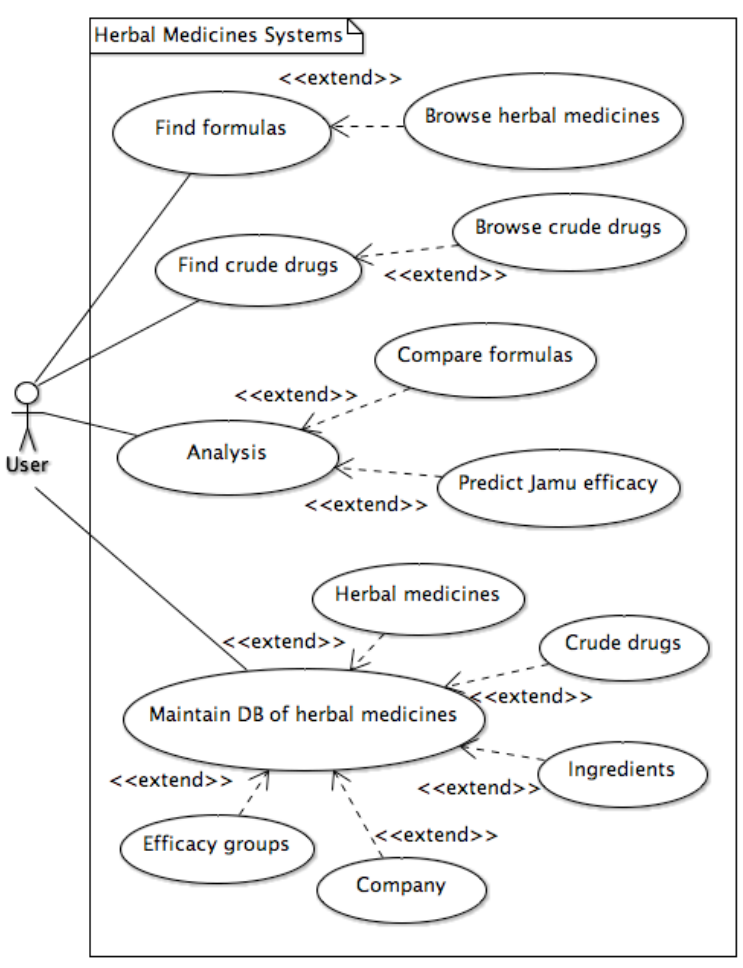

Fig. 3. Use case diagram of Herbal Medicine Systems

(246 and 777, respectively) kind of crude drugs. We also transformed some attributes from Kampo and Jamu databases to maintain its consistency and to satisfy the second normal form [30], e.g. split the data in the sid column of gakumei table into two or more rows. The rest of data warehouse pre-processing technique will be explained in the next sections.

Furthermore, we also designed the user interface of HMS application. The user interface of HMS is divided into three sections, i.e. header, body, and footer. In the header and body sections, it contains application title and main activities, i.e. input, output, or modify the data. In the footer section, there is a list of application features as follows: find herbal medicine formulas based on their name or efficacy ( $\left({ }^{-}\right)$, find crude drugs based on their name or effect $(-)$, analyze (compare herbal medicine formulas and predict Jamu efficacy, B), maintain the database of HMS ( (?).

\section{c. Implementation, integration and testing}

We implemented database of HMS from domain class diagram (Fig. 4) by using SQLiteStudio 3.0.6. Each class in the Fig. 4 becomes a two-dimensional table, where columns represent attributes and rows represent objects. The HMS database is composed of nine tables with the attributes in each table are equal to attributes of 


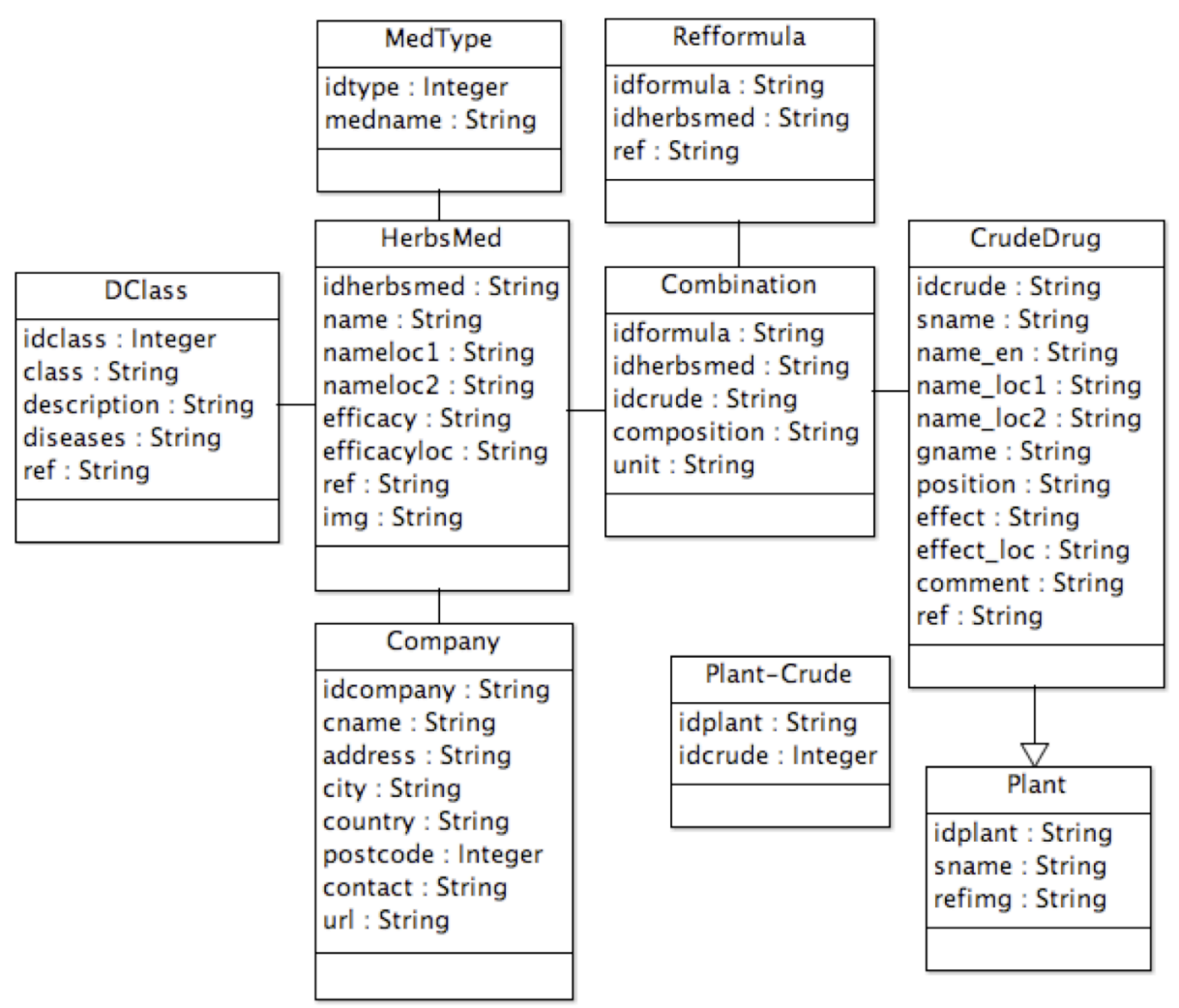

Fig. 4. Class diagram of Herbal Medicine Systems follows:

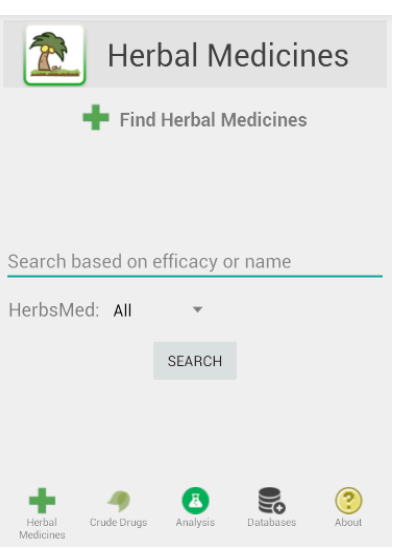

(a)
HMS domain class diagram. After the implementation of HMS database, the selected data from Kampo and Jamu databases were loaded into HMS database. In addition, we translated Kampo efficacies from Japanese to English to accommodate bilingual transaction processing. The tools used to develop the HMS application were as

- Scripting language: Java and XML

- Implementation tools: Android Studio Build \#AI-141.2168647

Fig. 5 shows some of the implementation results of HMS. The unit testing was done by using Black-box testing. Initially, we defined testing scenarios and

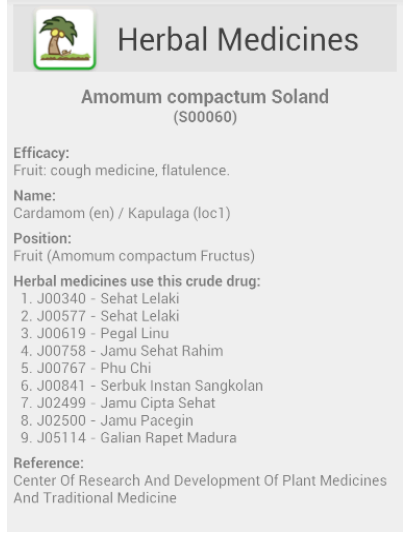

(b) compared the expected result to the actual result. The HMS application was tested by using Android Virtual Device (with specification 4.7" 768×1280 pixels, OS Android Jelly Bean) and Sony Xperia M2 dual D2302 (OS Android KitKat). The HMS application worked perfectly when we did the unit testing activities i.e. installation, loading the application and database, searching the herbal medicines and crude drugs, analyzing (comparing the herbal medicine formulas and prediction of Jamu efficacy), and maintaining the HMS database. Based on the system testing results, we concluded that the HMS conforms to the requirement definitions.

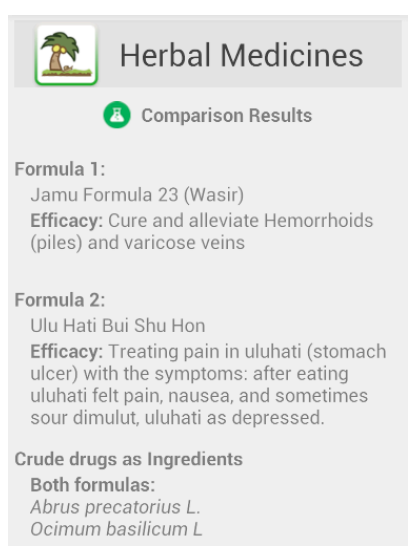

(c)

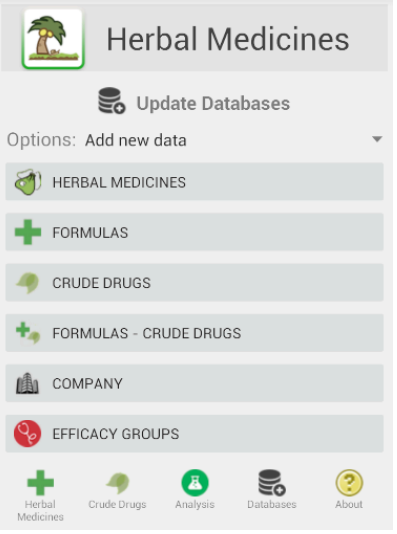

(d)
Fig. 5. The Herbal Medicine Systems: (a) find herbal medicines, (b) detail of crude drugs, (c) compare the recipes of herbal medicines, and (d) maintain the HMS database 


\section{d. Operation and maintenance}

Fig. 6 shows the main page of HMS application on Google Play store. Meanwhile, we have refreshed the HMS database with a new data collected from many references, i.e. the National Agency of Drug and Food Control (NADFC) of Indonesia, packages of herbal medicines, books of herbal medicine, the Internet and other related databases. An updated version of HMS database will be included in the latest update of the HMS application.

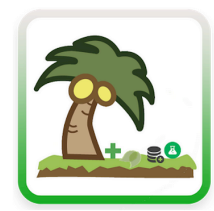

\section{Herbal Medicine Systems}

CSB NAIST Books \& Reference

$3+$

(1) This app is compatible with your device.

Fig. 6. The Herbal Medicine Systems' page on Google Play store

\section{e. Prediction of Jamu efficacy using Random Forest}

We implemented the efficacy prediction of mixing crude drugs by using $\mathrm{R}$ language. The prediction model was developed by $\mathrm{R}$ package named randomForest [31] and the user interface was developed by Shiny (http://shiny.rstudio.com/). The number of Jamu formulas used for generating the model is 3,012 , which are classified into 14 efficacy groups as follows: blood and lymph diseases (E1), cancers (E2), the digestive system (E3), female-specific diseases (E4), the heart and blood vessels (E5), diseases of the immune system (E6), male-specific diseases (E7), muscle and bone (E8), the nervous system (E9), nutritional and metabolic diseases (E10), respiratory diseases (E11), skin and connective tissue (E12), the urinary system (E13), and mental and behavioral disorders (E14). Moreover, the number of plants used as Jamu ingredients for prediction purposes is 465. Two important parameters while generating the model of Random Forest classifier are mtry and ntree. The tuneRF function was used to determine the best mtry value with the lowest OOB error, which is equal to 42 . In addition, the plots of OOB error and each efficacy group tend to be constant when the number of decision trees is larger than 500. Then, we set the ntree as 500. The classification result for each efficacy group is shown in Table 1. On an average, the Random Forest classifier can obtain accuracy around $90 \%$, with E3 class produce the largest accuracy. This feature might be useful for researchers to predict the efficacy of crude drug combinations for developing new drugs.
In the current version, the functionality of this feature is hosted in the server of Computational Systems Biology laboratory, NAIST. Initially, the HMS application will show the list of crude drugs. Then, user can select a combination of crude drugs, send a request to the server for efficacy prediction and can get the results back to his/her mobile phone.

Table 1. Prediction of Jamu efficacy according to efficacy-Jamu-plant relations

\begin{tabular}{cccc}
\hline $\begin{array}{c}\text { Efficacy } \\
\text { Groups }\end{array}$ & $\begin{array}{c}\text { Number of } \\
\text { Jamu }\end{array}$ & $\begin{array}{c}\text { Num. of correctly } \\
\text { classified }\end{array}$ & $\begin{array}{c}\text { Accuracy } \\
(\%)\end{array}$ \\
\hline E1 & 201 & 147 & 73.10 \\
E2 & 32 & 22 & 68.80 \\
E3 & 457 & 429 & 93.90 \\
E4 & 382 & 356 & 93.20 \\
E5 & 57 & 46 & 80.70 \\
E6 & 22 & 19 & 86.40 \\
E7 & 17 & 15 & 88.20 \\
E8 & 649 & 600 & 92.40 \\
E9 & 32 & 23 & 71.90 \\
E10 & 576 & 529 & 91.80 \\
E11 & 313 & 288 & 92.00 \\
E12 & 163 & 139 & 85.30 \\
E13 & 90 & 81 & 90.00 \\
E14 & 21 & 18 & 85.70 \\
Total & 3012 & 2712 & \\
\hline
\end{tabular}

\section{Conclusions}

The Herbal Medicine Systems (HMS) is a mobile application that provides information about various herbal medicines for disease treatment and promotes the use of herbal medicine. It is preloaded with 336 and 5,310 Japanese Kampo and Indonesian Jamu formulas respectively, taken from the KNApSAcK Family Databases. The HMS application mainly consists of four features i.e. find formulas/recipes based on their name and efficacy, find crude drugs based on their name and effect, analyze (compare herbal medicine formulas and predict the efficacy of crude drug combinations based on the formulation of Indonesian Jamu), and maintain database of HMS. Furthermore, the user of HMS application can modify the existing traditional medicines by adding other traditional medicine systems or new formulas. The efficacy prediction of crude drug combinations was developed by Random Forest classifier with a very high average accuracy.

The black-box testing results show that the functionality of HMS application conforms all functional requirements. The Herbal Medicine Systems application has been released at Google Play store, which can be downloaded and used freely. 


\section{References}

[1] R. Varpoorte, H. Kim, Y. Choi, Med. Aromat. Plants. Springer, Netherlands. (2006) 261-273.

[2] A. Furnham, Complement. Med. An Object. Apprais. Ed. by Ernst E. Oxford Butterworth-Heinemann. (1996).

[3] E. Ernst, BMJ Br. Med.J. 327 (2003) 881.

[4] A.T. Borchers, S. Sakai, G.L. Henderson, M.R. Harkey, C.L. Keen, J.S. Stern, et al., J. Ethnopharmacol. 73 (2000) 1-13.

[5] K. Watanabe, K. Matsuura, P. Gao, L. Hottenbacher, H. Tokunaga, K. Nishimura, et al., Altern. Med. (2011).

[6] F.M. Afendi, L.K. Darusman, A. Hirai, M. Altaf-Ul-Amin, H. Takahashi, K. Nakamura, et al., Proc. IEEE Int. Conf. Data Mining, ICDM, IEEE, (2010) 661-668.

[7] F.M. Afendi, L.K. Darusman, A.H. Morita, M. Altaf-Ul-Amin, H. Takahashi, K. Nakamura, et al., Curr. Comput. Aided. Drug Des. 9 (2013) 46-59.

[8] S.H. Wijaya, H. Husnawati, F.M. Afendi, I. Batubara, L.K. Darusman, M. Altaf-Ul-Amin, et al., Biomed Res. Int. (2014) 1-15.

[9] K.C. Huang, The pharmacology of Chinese herbs, CRC press, 1998.

[10] R. Yuan, Y. Lin, Pharmacol. Ther. 86 (2000) 191-198.

[11] B. Patwardhan, D. Warude, P. Pushpangadan, N. Bhatt, Evidence-Based Complement. Altern. Med. 2 (2005) 465-473.

[12] B. Patwardhan, A.D.B. Vaidya, M. Chorghade, Curr. Sci. 86 (2004) 789-799.

[13] I. Sommerville, Software Engineering, $9^{\text {th }}$ ed., Pearson, 2010.

[14] X. Zhu, H. Zhao, Appl. Econ. Bus. Dev., Springer Berlin Heidelberg, (2011) 170-176.

[15] N.M.A. Munassar, A. Govardhan, Int. J. Comput.
Sci. Issues. 7 (2010) 94-101.

[16] M. Khalifa, J.M. Verner, IEEE Trans. Eng. Manag. 47 (2000) 360-369.

[17] S. Balaji, M. Murugaiyan, Int. J. Inf. Technol. Bus. Manag. 2 (2012) 26-30.

[18] F.M. Afendi, T. Okada, M. Yamazaki, A. Hirai-Morita, Y. Nakamura, K. Nakamura, et al., Plant Cell Physiol. 53 (2012) e1.1-12. doi:10.1093/pcp/pcr165.

[19] L. Breiman, Mach. Learn. 45 (2001) 5-32.

[20] R. Díaz-Uriarte, S.A. De Andres, BMC Bioinformatics. 7 (2006) 3.

[21] L. Yang, N. Boushehrinejadmoradi, P. Roy, V. Ganapathy, L. Iftode, Proc. Second ACM Work. Secur. Priv. Smartphones Mob. Devices, (2012) 21-26.

[22] S.D. Burd, R.B. Jackson, J.W. Satzinger, Systems Analysis and Design in a Changing World, 2004.

[23] B.C. Zapata, Android Studio Application Development, Packt Publishing Ltd, 2013.

[24] B.C. Zapata, Android Studio Essentials, Packt Publishing Ltd, 2015.

[25] S. Haldar, SQLite Database System Design and Implementation, Sibsankar Haldar, 2015.

[26] J. Han, M. Kamber, J. Pei, Data mining: concepts and techniques: concepts and techniques, Elsevier, 2011.

[27] R. Jiang, W. Tang, X. Wu, W. Fu, BMC Bioinformatics. 10 (2009) S65.

[28] B.H. Menze, B.M. Kelm, R. Masuch, U. Himmelreich, P. Bachert, W. Petrich, et al., BMC Bioinformatics. 10 (2009) 213.

[29] R.O. Duda, P.E. Hart, D.G. Stork, Pattern classification, John Wiley \& Sons, 2012.

[30] T.M. Connolly, C.E. Begg, Database systems: a practical approach to design, implementation, and management, Pearson Education, 2005.

[31] A. Liaw, M. Wiener, R News. 2 (2002) 18-22. 